\title{
Effects of thickness on electronic structure of titanium thin films
}

\author{
GÜVENÇ AKGÜL \\ Bor Vocational School, Nigde University, 51700 Nigde, Turkey
}

MS received 26 August 2012

\begin{abstract}
Effects of thickness on the electronic structure of e-beam evaporated thin titanium films were studied using near-edge X-ray absorption fine structure (NEXAFS) technique at titanium $L_{2,3}$ edge in total electron yield (TEY) mode and transmission yield mode. Thickness dependence of $L_{2,3}$ branching ratio (BR) of titanium was investigated and it was found that BR below $3.5 \mathrm{~nm}$ shows a strong dependence on film thickness. Mean electron escape depth $(\lambda)$ in titanium, an important parameter for surface applications, was determined to be $\lambda=2.6 \pm$ $0 \cdot 1 \mathrm{~nm}$ using $L_{2,3}$ resonance intensity variation as a function of film thickness. The average $L_{3} / L_{2}$ white line intensity ratio of titanium was obtained as 0.89 from the ratio of amplitudes of each $L_{3}$ and $L_{2}$ peaks and 0.66 from the integrated area under each $L_{3}$ and $L_{2}$ peaks. In addition, a theoretical calculation for pure titanium was presented for comparison with experimental data.
\end{abstract}

Keywords. NEXAFS; titanium; branching ratio; electron escape depth; thin films.

\section{Introduction}

Electronic properties are important for fundamental understanding of the macroscopic and microscopic behaviour of materials (Hähner 2006). In recent years, near-edge Xray absorption fine structure (NEXAFS) spectroscopy has become a powerful experimental technique to get electronic structural information of elements or molecules (Vergara et al 1999; Stoyanov et al 2007). Particularly, L-edge NEXAFS provides a sensitive fingerprint of the electronic states of magnetic $3 d$ transition metals (Chakarian et al 1998; Nakajima et al 1999). NEXAFS measurements are generally performed in total electron yield (TEY) mode by measuring the total electron number per incident photon emitted from the material as a function of photon energy $(\hbar \omega)$ and in transmission mode by measuring the intensity of X-ray beam before and after transmission through a thin film material. NEXAFS spectrum exhibits sharp absorption edges which occur at energies at which excitation of particular core level becomes energetically allowed. The energies of absorption edges are characteristic of each element. For $L_{2,3}$ edges, the dipole-allowed transition that involves the excitation of a transition metal $2 p$ electron to unfilled metal $3 d$ orbital provides a basis for interpretation of the measured spectra, in other words, the absorption edges (Winick 1980; Koningsberger and Prins 1988; Stöhr 1992; Sakurai 1994). The spectral features in the dipole-allowed transitions are intense and dependent on the oxidation state, spin state and local environment (Van der Laan and Kirkman 1992).

The large spin-orbit interaction of $2 p$ core hole splits NEXAFS spectrum into $L_{3}\left(2 p_{3 / 2}\right)$ and $L_{2}\left(2 p_{1 / 2}\right)$ levels. This interaction has an important influence on the branching

guvencakgul@gmail.com ratio (BR) between $L_{3}$ and $L_{2}$ peaks. BR can be defined as the intensity ratio $\mathrm{I}\left(L_{3}\right) /\left[\mathrm{I}\left(L_{2}\right)+\mathrm{I}\left(L_{3}\right)\right]$, where $I\left(L_{3}\right)$ and $I\left(L_{2}\right)$ are the measured intensity of the peaks of $j=3 / 2$ and $j=1 / 2$ components, respectively. Although, the relative intensity of these two peaks would be $2: 1$ according to one electron band structure calculation, it can be affected by angular momentum coupling and strongly deviates from 2:1, the so-called anomalous BR. Anomalous BR has been the subject of many studies in the literature (Pease et al 2001; Kurmaev et al 2005).

$3 d$ transition metals and their compounds possess unique physical and chemical properties, resulting in their applications as advanced materials. Especially, thin film structures involving $3 d$ metals are probably the most interesting and widely used materials in technology. Since, the development of new materials necessitates the determination of their basic parameters, it is crucial to determine their electronic structures. Recently, the electronic structure of titanium and its oxides has been the subject to a wide range of studies due to its technological applications from catalysis to aircraft construction. In addition, titanium compounds are known to be the best, biocompatible materials to be used for orthopedic and dental (Lee et al 1991; Hanawa and Ota 1992; Müller and Hauert 1996; Vaquila et al 1993, 1997; Oviedo 1993). The technological importance makes titanium an attractive material to be paid great attention.

Surface properties play an important role since, they determine the interaction of an object with its environment. The basic knowledge of the structure and electronic characteristic of the surface of a material is therefore, crucial for many applications. The escape depth of the emitted electrons $(\lambda)$ is one of the important parameters for thin film applications and it is mainly the characteristic of surface properties of the sample. Thus, the precise estimation of this parameter 
is especially important to determine sensitivity of the surface. Electron escape depth measurements are generally estimated by photo-emission and Auger electrons only for the specific samples (Lindau and Spicer 1974; Tokutaka et al 1985). In the last several years, there have been many reports that involve estimating the escape depth of the emitted electrons (Chakarian et al 1998; Nakajima et al 1999; Gota et al 2000; Ufuktepe et al 2005; Akgül et al 2008). However, no comparable work has been done on the electron escape depth in titanium transition metal. This is the first study, to the best of our knowledge, to calculate the electron escape depth for pure titanium. Obtaining results on the atomic structures of titanium can be a key requirement for the development of TM based applications.

This study is focused on the effects of thickness on the electronic properties of titanium thin films to estimate the accurate electron escape depth from NEXAFS spectra. The results of measurements on TEY and transmission yield NEXAFS from titanium metal thin films with different thicknesses at $L_{2,3}$ absorption edges are presented here to calculate this parameter. Thickness dependence of titanium $L_{2,3}$ branching ratio is also investigated. In addition to NEXAFS measurements, a theoretical calculation is given for the purpose of comparison with experimental data.

\section{Experimental}

NEXAFS experiments were performed at Beamline 8.2 at the Stanford Synchrotron Radiation Laboratory (SSRL). The beamline 8.2 is a bending magnet dedicated to probe a wide range of core levels using photoemission, photo-absorption, NEXAFS and has an energy range from 100-1300 eV. Total electron yield and transmission spectra were collected at room temperature. Titanium thin films were grown by ebeam evaporation on $\mathrm{SiN}$ coated Si wafers. Soft X-ray transparent $100 \mathrm{~nm}$ thick SiN window was at the middle of $(4 \times$ $1.5 \mathrm{~mm}$ ) square $\mathrm{Si}$ wafer that allows performing transmission measurements. For total electron yield measurements, titanium films were grown on $200 \mu \mathrm{m} \mathrm{Si}$ wafer, which was the frame of SiN window. Thickness of titanium layers ranging from 1-10 $\mathrm{nm}$ was determined with a quartz crystal thin film monitor. Base pressure of the sample preparation chamber was $1 \times 10^{-10}$ torr and below $1 \times 10^{-9}$ torr during evaporation. The substrates were mounted on sample holders, which could be transferred without breaking vacuum between film growth and analysis chamber. To perform transmission measurements, the attenuation of X-ray flux was determined by monitoring the incident photon flux with highly transmittive gold grid and measuring the transmitted photon flux with a silicon photodiode, that was mounted just behind SiN membrane window. The photodiode was operated without a bias voltage and shielded from all the light sources besides incoming photons passing through the sample. In order to eliminate effect of substrate from absorption spectrum, a photo-absorption spectrum of $\mathrm{SiN}$ substrate itself was collected just before deposition of titanium. In order to record TEY spectra, TEY signal of the sample was monitored by drain current through the wire connecting the otherwise electrically insulated sample to ground and the number of incoming photons by TEY signal of $80 \%$ transmissive Au net. The ratio of the sample signal and incoming photon flux was then the TEY spectrum of interest for measuring sample absorption coefficient.

\section{Results and discussion}

Figure 1 shows normalized NEXAFS spectra of titanium thin films at $L_{2,3}$ region with varying thicknesses ranging from 1-10 nm in TEY mode. Titanium thicknesses in nanometer (nm) units are given beside each curve. All the spectra were collected at room temperature. In each spectrum, TEY signal before the onset of $L_{3}$ edge at $446 \mathrm{eV}$ was set to zero. The intensity of TEY signal thus reflects solely to the contribution of titanium $L_{2,3}$ levels to the absorption coefficient. The main features in each spectrum are two maxima at 456.6 and $462.3 \mathrm{eV}$ corresponding to edges of $L_{3}$ and $L_{2}$, respectively. These two peaks can be explained by the electrostatic interaction between core-hole and valence electrons and the initial state-spin orbit splitting. The energy separation between two peaks is measured as $5.7 \mathrm{eV}$ due to the spin-orbit interaction of the titanium $2 p$ core level (Soriano et al 1993; Lusvardi et al 1998; Mazza et al 2008; Lau et al 2008; http://xdb.lbl.gov/Section1/Periodic_Table/ Ti_Web_data.htm). The splitting between titanium $L_{3}$ and $L_{2}$

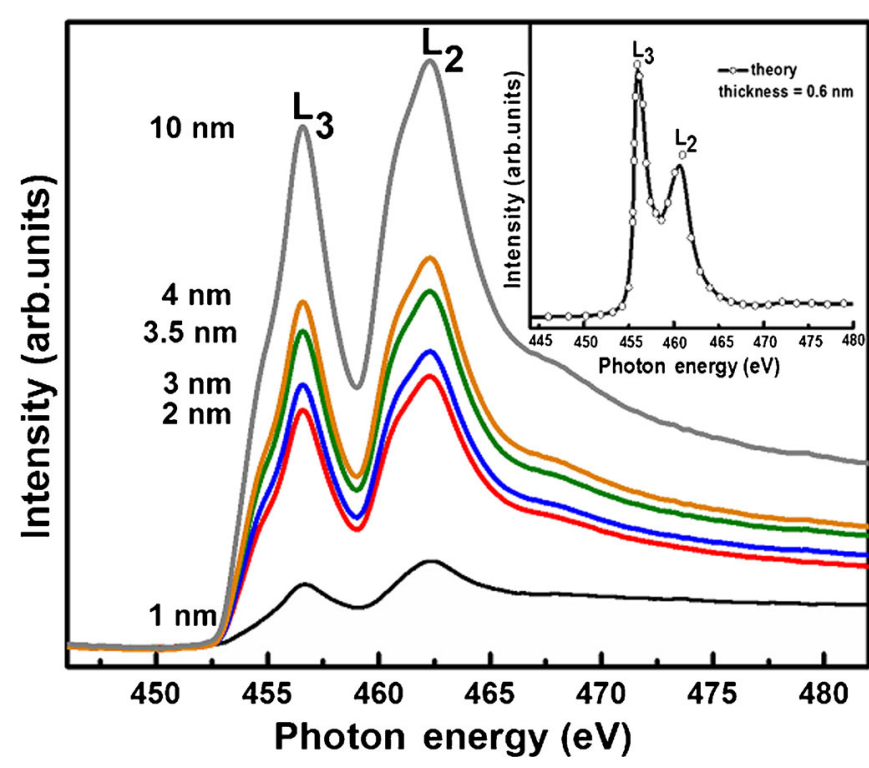

Figure 1. Normalized total electron yield (TEY) spectra of titanium films with different film thicknesses. Titanium thicknesses in nanometer $(\mathrm{nm})$ units are given beside each curve. In each spectrum, TEY signal before onset of $L_{3}$ edge at $446 \mathrm{eV}$ was set to zero. Inset shows theoretical calculation for titanium thin film with a thickness of $0.6 \mathrm{~nm}$. 


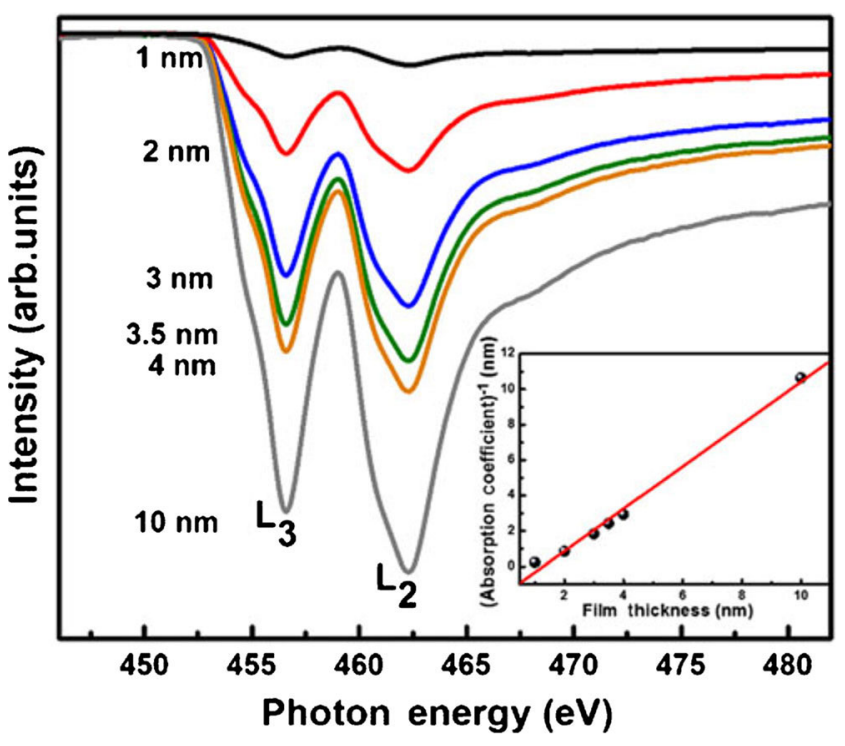

Figure 2. Normalized transmission yield spectra of titanium films with different thicknesses in vicinity of $2 p$ thresholds are taken at normal incidence. Titanium thicknesses in nanometer $(\mathrm{nm})$ units are given beside each curve. Inset shows plot of absorption coefficient obtained for different thicknesses. Solid line is a linear curve fit which shows transmission detection and normalization was linear.

edges are reproduced by theoretical calculation. The inset in figure 1 shows theoretically calculated spectrum for titanium film with a thickness of $0.6 \mathrm{~nm}$ for comparison with experimental data. FEFF 8.2, which is a self-consistent real space multiple-scattering code for simultaneous calculations of X-ray absorption spectra including polarization dependence and local field corrections were used for theoretical calculation of $L_{3}$ and $L_{2}$ edges (Ravel 2001). The experimental NEXAFS spectra presented for titanium at $L_{2,3}$ edges can be adequately described by theory. Different intensities of $L_{3}$ and $L_{2}$ edges in NEXAFS spectra for the experiment and theory can be explained by $2 p-3 d$ core-hole correlation effects, which are observed strongly in the early $3 d$ transition metals (Andreas 2003). These effects may not be included in FEFF 8.2 program and it can cause inconsistency between theory and experimental spectrum.

TEY and transmission yield spectra have been recorded simultaneously. Figure 2 shows room temperature NEXAFS spectra of titanium thin films at $L_{2,3}$ edges, which vary in thickness from 1-10 nm, in transmission mode. A similarity between both spectra of figures 1 and 2 is observed. The main peaks are the typical structure of titanium $L$-edge (Soriano et al 1993; Lusvardi et al 1998). No detectable energy shift of $L_{2,3}$ edges in the film growing process has been noticed but the intensity of both peaks were changed as a function of film thickness. The absorption coefficient obtained for different thicknesses was plotted in the inset of figure 2 to show transmission detection and normalization was indeed linear.

In X-ray spectral region, photoelectric absorption is the dominant interaction mechanism between incoming photon radiation and material. For the photon intensity, the normal decay will be assumed, so that

$$
I(\hbar \omega)=I_{\mathrm{o}}(\hbar \omega) e^{-\sigma(\hbar \omega) d},
$$

where $I_{\mathrm{o}}$ is the incident intensity, $\sigma$ the absorption coefficient and $d$ the depth below the surface. The product $\sigma(\hbar \omega) d$ is a quantity proportional to the absorbance and can be obtained by inverting (1). X-ray absorption spectrum is a measurement of the absorption coefficient of a sample, $\sigma(\hbar \omega)$ or of a quantity to which it is directly proportional, as a function of energy of the impinging photons, $\hbar \omega$. The overall absorption coefficient of a sample is related to the atomic concentrations $\left(\rho_{\mathrm{i}}\right)$ and absorption cross-sections $\left(\alpha_{\mathrm{i}}(\hbar \omega)\right)$ of the constituent atoms and is given by the expression

$$
\sigma(\hbar \omega)=\sum_{\mathrm{i}} \rho_{\mathrm{i}} a_{\mathrm{i}}(\hbar \omega) .
$$

Titanium signal in figures 1 and 2 increases rapidly with increasing film thickness as expected. To quantify this observation, the intensity of $L_{3}$ and $L_{2}$ edges vs film thickness was plotted. In both cases, difference between the respective yield signals and the preedge intensity at the photon energy of $446 \mathrm{eV}$ was considered. Figure 3 represents measured values of relative peak heights of $L_{3}$ and $L_{2}$ resonances in TEY mode as a function of titanium film thickness. In the case of a thin film, TEY intensity can be written as (Thole and Van der Laan 1988)

$$
I(d)=I_{\infty}\left[1-e^{-d / \lambda}\right],
$$

where $d$ is the film thickness, $I_{\infty}$ the maximum TEY signal as measured for the infinitely thick bulk material and $\lambda$ the

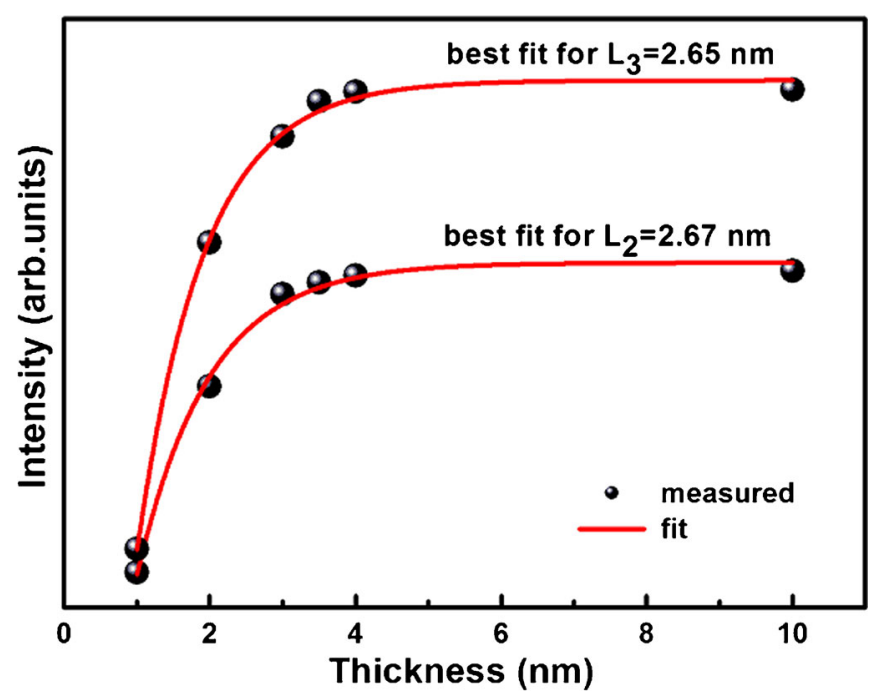

Figure 3. Normalized peak heights of $L_{3}$ and $L_{2}$ resonance as a function of titanium film thickness. Solid curves are result of fitting equation $y=A\left[1-e^{-b d}\right]$ to respective film thickness dependencies. Best fit using mean electron escape depth of emitted electrons as a free parameter was obtained for 2.65 and $2.67 \mathrm{~nm}$. 
electron escape depth. The electron escape depth is a quantity which determines the surface sensitivity of the material of interest and is unknown for most of the materials. The escape depth of NEXAFS measurements in TEY mode is generally estimated by theoretical calculations with the use of a universal curve, which is related to the diffusion length of the electrons and this quantity is difficult to calculate. TEY spectra offer an advantage to determine the electron escape depth of a material experimentally. In order to estimate escape depth through titanium thin films, the curve fitting is performed. The solid curves in figure 3 are the results of fitting (3) to the respective film thickness dependencies. The best fit using the electron escape depth as a free parameter was found for the values of $\lambda=2.65$ and $2.67 \mathrm{~nm}$ for $L_{3}$ and $L_{2}$, respectively. The maximum electron escape depth of core level binding energies ranging from $77-929 \mathrm{eV}$ was measured to be between 15 and $141 \AA$ by Frazer et al (2003) but no data is available in the literature for comparison. To the best of our knowledge, the electron escape depth at $L_{2}$ and $L_{3}$ edges is first to be measured for $3 d$ titanium metal.

According to Thole and Van der Laan (1988), an electrostatic interaction between $d$ electron and valence hole and also spin-orbit interaction of $d$ electrons affect the branching ratio (BR). In this study, experimental BR intensity of titanium thin films is also investigated. Generally, $L_{3}$ and $L_{2}$ NEXAFS spectra include contributions arising from $2 p-3 d$ transitions as well as excitations from $2 p$ core level to continuum states. In order to eliminate the transitions into continuum states, in other words, to isolate the excitation spectra only from the inner-shell $2 p$ core level to the bound $3 d$ state, it is necessary to remove the background component. Figure 4(a) represents normalized and background corrected TEY spectra of titanium thin films with varying film thicknesses. Two step-like functions aligned at the maxima of $L_{3}$ and $L_{2}$ lines with relative heights of $2: 1$, which is the expected intensity ratio for transitions into $L_{3}$ and $L_{2}$ edges, were used for the background subtraction of NEXAFS spectra. Because, the area of $L_{3}$ and $L_{2}$ lines would be the correct measure of their intensity, which is proportional to the oscillator strength of $2 p-3 d$ spin-orbit interaction, $L_{3}$ and $L_{2}$ peak areas were used to derive the experimental $L_{3}-L_{2}$ BR intensity for titanium thin film. Figure 4(b) presents measured branching ratio $\left(I\left(L_{3}\right) /\left[I\left(L_{2}\right)+I\left(L_{3}\right)\right]\right)$ of the background corrected integrated intensities at $L_{2,3}$ edge as a function of titanium film thickness. It can be seen from figure 4(b) that BR changes significantly from 0.69 to 0.60 , as the film thickness increases from 1-10 $\mathrm{nm}$. The values measured here are consistent with the values obtained from previous study, which are between 0.41 and 0.80 in $3 d$ transition metal series (Van der Laan 1989). BR of titanium films below $3.5 \mathrm{~nm}$ shows a strong dependence on film thickness. On the other hand, data points above this critical thickness at $4-10 \mathrm{~nm}$ in figure 4(b) reveals that BR is almost constant and close to the bulk value determined for titanium which is $\sim 0.6$ (Thole and Van der Laan 1988). The thickness dependent variation of the experimental $L_{2,3}$ BR in titanium can be explained by screening of $p-d$ electrostatic interaction with increased delocalized valence electrons in thicker films. Also, the variation of BR with increasing thickness is also proportional to the strength of spin-orbit splitting. From TEY spectra as shown in figure 4(a), $2 p$ spin-orbit splitting of the samples was measured and it was found that the splitting is not significantly changing with increasing film thickness for titanium. This finding clearly indicates that the variation of BR rather originated by core-hole screening with increased electron delocalization in thicker films than by spin-orbit splitting. The plot of BR vs inverse of film thickness is also shown in the inset of figure 4(b). Since, the solid line which is a linear curve fit to experimental data assumes an ideal layer-bylayer growth, growth mode of titanium thin films show good agreement with layer-by-layer model.

Using NEXAFS spectra in TEY mode, the average value of $L_{3} / L_{2}$ ratio of titanium has been measured as 0.66 and 0.89 , from the integrated peak area and peak intensity, respectively. These measurements confirm the results of Mazza et al (2008) and Leapman Grunes (1980).
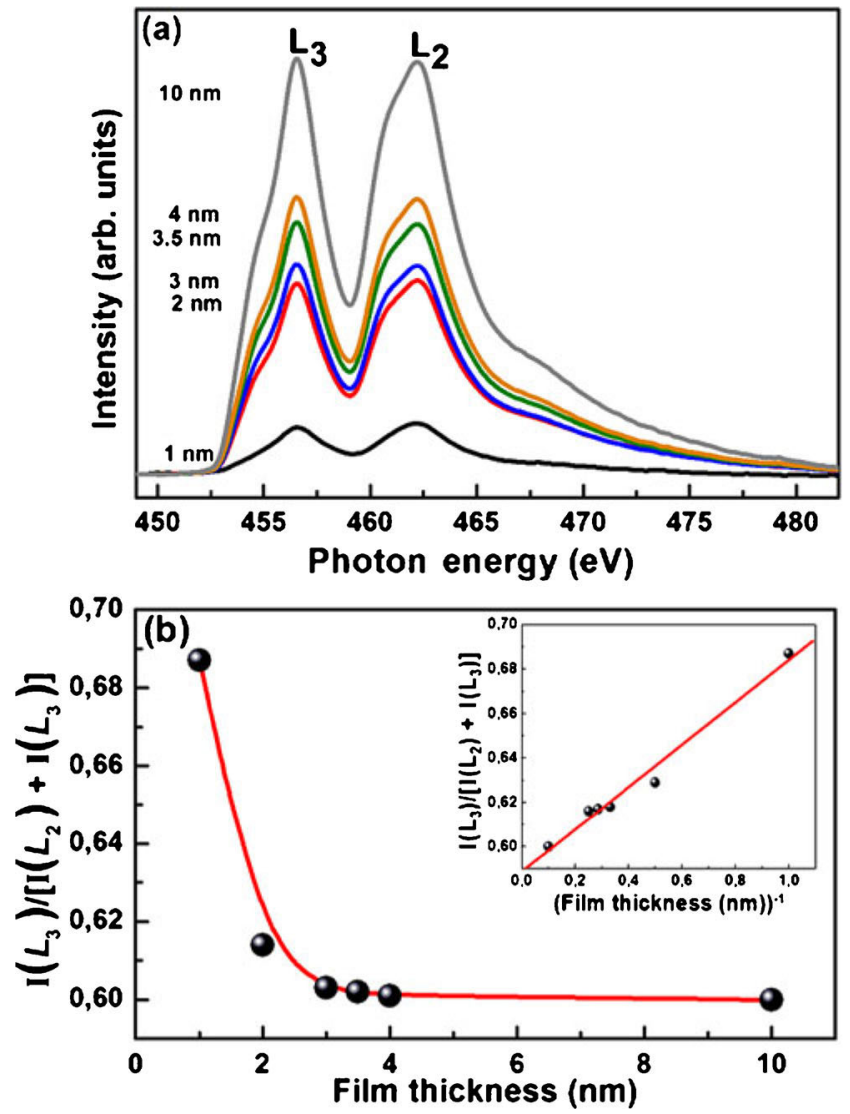

Figure 4. (a) Normalized and background corrected total electron yield (TEY) spectra of titanium films with different film thicknesses and (b) branching ratio (BR) of background corrected intensities at $L_{2,3}$ edge as a function of titanium film thickness. Drawn line is only a guide to the eye. Inset shows plot of BR intensity vs inverse of film thickness. 


\section{Conclusions}

To summarize, titanium thin films with thickness ranging from 1-10 $\mathrm{nm}$ were prepared by e-beam evaporation method. TEY and transmission yield at titanium $L_{2,3}$ edge with varying titanium film thicknesses were studied. Two maxima, at 456.6 and $462.3 \mathrm{eV}$, corresponding to $L_{3}$ and $L_{2}$ edges of titanium were observed in both TEY and transmission yield spectra for all thicknesses. The variation of experimental titanium BR with film thickness was presented. In addition, the average value of $L_{3} / L_{2}$ ratio of titanium was calculated to be 0.66 from integrated area and 0.89 from peak intensity, respectively. TEY measurements were used to derive electron escape depth at the titanium $L$-edge and the average value $\lambda=2.6 \pm 0.1 \mathrm{~nm}$ was found. To the best of our knowledge, no data on electron escape depth of titanium is available in the literature for comparison. In this paper, electron escape depth at $L_{2,3}$ edge is first to be determined for titanium thin film. Therefore, present study may complete the missing information of effects of thickness on the electronic structure of titanium thin film.

It has also been shown that NEXAFS is a suitable technique to investigate the electronic structure of the investigated materials and to determine the electron escape depth on their surfaces. Measurements on other $3 d$ transition metals are in progress in order to estimate the effect of $3 d$ electron occupation on the electron escape depth of the emitted electrons and branching ratio.

\section{Acknowledgements}

The author is grateful to Profs Piero Pianetta, Herman Winick and staff at the Stanford Synchrotron Radiation Lightsource (SSRL) for their excellent support, where NEXAFS experiments have been carried out. (SSRL) is supported by the Department of Energy (DOE), Office of Basic Energy Science.

\section{References}

Akgül G, Aksoy F, Bozduman A, Ozkendir O M, Ufuktepe Y and Lüning J 2008 Thin Solid Films 5171000
Andreas S 2003 thesis ISBN: 3-89825-779-7

Chakarian V, Idzerda Y U and Chen C T 1998 Phys. Rev. B 575312

Frazer B H, Gilbert B, Sonderegger B R and De Satsio G 2003 Surf. Sci. $\mathbf{5 3 7} 161$

Gota S, Gautier Soyer M and Sacchi M 2000 Phys. Rev. B 624187

Hanawa T and Ota M 1992 Appl. Surf. Sci. 55269

Hähner G 2006 Chem.Soc. Rev. 351244

http://xdb.lbl.gov/Section1/Periodic_Table/Ti_Web_data.htm

Koningsberger D C and Prins R 1988 X-ray absorption (New Jersey: John Wiley and Sons)

Kurmaev E Z, Ankudinov A L, Rehr J J, Finkelstein L D, Karimov P F and Moewes A 2005 J. Electron Spectrosc. Relat. Phenom. 1481

Lau J T, Rittmann J, Zamudio Bayer V, Vogel M, Hirsch K, Klar Ph, Lofink F and Möller T 2008 Phys. Rev. Lett. 101153401

Leapman R D and Grunes L A 1980 Phys. Rev. Lett. 45397

Lee P A, Stork K E, Maschhoff B L, Nebesny K W and Armstrong N R 1991 Surf. Interf. Anal. 1748

Lindau I and Spicer W E 1974 J Electron. Spectrosc. Relat. Phenom. 3409

Lusvardi V S, Barteau M A, Chen J G, Eng J, Frühberger B and Teplyakov A 1998 Surf. Sci. 397237

Mazza T, Piseri P, Bongiorno G, Ravagnan L, Amati M, Devetta M, Lenardi C and Coreno M 2008 Appl. Phys. A 92463

Müller U and Hauert R 1996 Thin Solid Films 290323

Nakajima R, Stöhr J and Idzerda Y U 1999 Phys. Rev. B 596421

Oviedo C 1993 J. Phys. Condens. Matter A 5153

Pease D M, Fasihuddin A, Daniel M and Budnick J I 2001 Ultramicroscopy $\mathbf{8 8} 1$

Ravel B 2001 J. Synchroton Rad. 8314

Sakurai J J 1994 Modern quantum mechanics (New York: Addison Wesley)

Soriano L, Abbate M, Vogel J, Fuggle J C, Fernández A, González Elipe A R, Sacchi M and Sanz J M 1993 Surf. Sci. 290427

Stoyanov E, Langenhorst F and Steinle Neumann G 2007 Am. Miner. 925773

Stöhr J 1992 Nexafs spectroscopy (Heidelberg: Springer) 25

Thole B T and Van der Laan G 1988 Phys. Rev. B 383158

Tokutaka H, Nishimori K and Hayashi H 1985 Surf. Sci. 149349

Ufuktepe Y, Akgül G and Lüning J 2005 J. Alloys Compds 401193

Van der Laan G 1989 Physica B $\mathbf{1 5 8} 395$

Van der Laan G and Kirkman I W 1992 J. Phys. Condens. Matter 4 4189

Vaquila I, Passeggi M C G and Ferrón J 1993 Surf. Sci. 292 L795

Vaquila I, Passeggi M C G and Ferrón J 1997 Phys. Rev. B 5513925

Vergara L I, Vaquila I and Ferron J 1999 Appl. Surf. Sci. 151129

Winick H 1980 Synchrotron radiation research (New York: Plenum Press) 